\title{
Oxidative photoreceptor cell damage in autoimmune uveitis
}

\author{
Angeline M. Nguyen • Narsing A. Rao
}

Received: 12 August 2010 / Accepted: 16 September 2010/Published online: 30 October 2010

(C) The Author(s) 2010. This article is published with open access at Springerlink.com

\begin{abstract}
Uveitis comprises an extensive array of intraocular inflammatory diseases and often results in irreversible visual loss. Experimental autoimmune uveitis (EAU) is an animal model used to study human uveitis. Both innate and adaptive immune responses are known to mediate retinal damage in EAU. The innate immune response occurs first with activation of toll-like receptors which upregulate inflammatory cytokines, leading to oxidative stress; subsequently, the adaptive immune response results in inflammatory cytokine upregulation and mitochondrial oxidative stress. In early EAU, mitochondrial DNA is damaged before inflammatory cellular infiltration and alters mitochondrial protein levels and the functions of mitochondria in AU. Our recent study confirms the importance of TLR4 in the generation of inflammatory cytokines, initiation of oxidative DNA damage, and induction of mitochondrial oxidative stress. Like EAU, sympathetic ophthalmia also results in photoreceptor mitochondrial oxidative damage. Agents that prevent mitochondrial oxidative stress and photoreceptor apoptosis may help prevent retinal damage and preserve vision in uveitis.
\end{abstract}

Keywords Experimental autoimmune uveitis - Sympathetic ophthalmia - TLR4 - Complete Freund's adjuvant .

Mitochondrial oxidative stress

\section{Introduction}

Uveitis is one of the major causes of blindness due primarily to retinal tissue damage, especially retinal photoreceptor

A. M. Nguyen • N. A. Rao $(\bowtie)$

Doheny Eye Institute, University of Southern California,

1450 San Pablo St. DVRC Room 211,

Los Angeles, CA 90033, USA

e-mail: nrao@usc.edu degeneration, caused by the release of cytokines and various other chemical mediators such as oxygen metabolites [1]. Experimental autoimmune uveitis (EAU) is an animal model used extensively to study the developmental mechanisms behind human uveitis. Uveitis is induced in mice and rats for the autoimmune disease model. In the mice models, EAU is induced by injecting interphotoreceptor retinoid-binding protein (IRBP) emulsified in complete Freund's adjuvant (CFA) supplemented with Mycobacterium tuberculosis strain H37RA. Bordetella pertussis toxin (PTX) is included as an additional adjuvant to CFA to increase the susceptibility of resistant strains; however, in some sensitive strains, such as B10RIII mice, PTX injection is not necessary [2]. Usually, the emulsion is injected subcutaneously, divided between the base of the tail and both thighs [3, 4]. In Lewis rats, the uveitis is induced by a hind foot-pad injection of bovine S-antigen or IRBP in CFA containing heat-killed M. tuberculosis strain H37RA [5-7]. In both mice and rats, the EAU is characterized by two phases, early EAU and amplified EAU. Early EAU is defined as the phase prior to day 7 postimmunization before infiltration of inflammatory cells into the retina and/or urea, whereas amplified EAU occurs after day 7, with a peak inflammation on day 14 (Fig. 1) revealing heavy infiltration and inflammatory cells in the retina and uvea.

A well-recognized mechanism of EAU development is the $\mathrm{T}$ cell-mediated adaptive immune response; however, recent reports show that the innate immune response also plays a crucial role in the initiation of EAU $[4,8,9]$. Both the adaptive and innate immune responses mediate retinal damage; the innate immune response occurs first with tolllike receptors upregulating inflammatory cytokines which leads to the induction of oxidative stress; the adaptive immune response occurs on a cellular level, but also results in inflammatory cytokine upregulation and oxidative stress (Fig. 2). 
EAU DAY 5

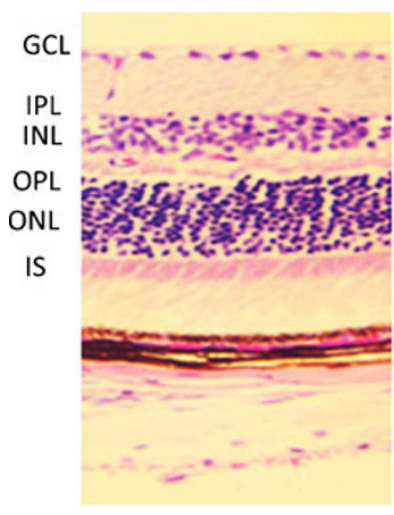

EAU DAY 14

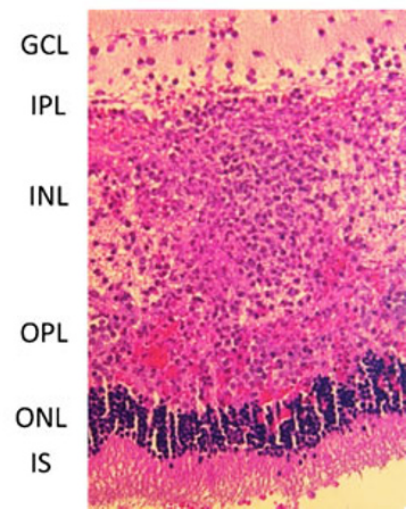

Fig. 1 Morphologic changes can be seen throughout experimental autoimmune uveoretinitis (EAU). Photomicrographs showing hematoxylin and eosin-stained sections of mice (B10RIII) retinas reveal that during early EAU (day 5 postimmunization), retinas remain intact; whereas at the peak of inflammation, during the amplification phase of EAU (day 14), macrophage and other inflammatory cell infiltration is prominent and the retinal structure is no longer well-preserved

The adaptive immune response has been widely studied in EAU. In previous years, EAU-related retinal damage was attributed to blood-borne activated macrophages and polymorchonuclear leukocytes, which release a variety of cytotoxic agents and free radicals, including, superoxide, hydrogen peroxide, and inducible nitric oxide synthase (iNOS) and related oxidant, peroxynitrite $\left(\mathrm{ONOO}^{-}\right)$in the retina, resulting in oxidative stress $[1,10-12]$. The oxidative stress primarily takes place in the mitochondria during the early phase, whereas nuclear oxidative damage is observed during the amplification phase of EAU. Mitochondrial proteins such as cytochrome $\mathrm{C}$ and mitochondrial import stimulation factor are the primary targets for inactivation by $\mathrm{ONOO}^{-}$which results in mitochondrial oxidative stress [6]. The oxidants and free radicals initiate retinal lipid peroxidation, causing the formation of hydroperoxides and their resultant chemotactic activity amplifies the inflammatory processes [13]. Localization of hydroperoxide-derived cellular carbonyls and cellular modification by tyrosine nitration were found concentrated in the photoreceptor layers at the height of inflammation during the amplified phase of EAU, postimmunization day 14 [10, 11, 14]. Macrophages typically infiltrate the retina during this amplification phase of EAU [6, 15]. In addition to macrophages, retinal microglia also exhibit phagocytic functions; once activated, microglia migrate to the outer retina and release the pathogenic factors $\mathrm{ONOO}^{-}$and tumor necrosis factor- $\alpha$ (TNF- $\left.\alpha\right)$ [15]. In EAU, microglial migration toward the photoreceptors may be mediated by the lipid peroxidation product decosahexaenoic acid hydroperoxide (22:6 HP) formed in the photoreceptors, and these migrating cells could help modulate inflammation [16]. However, contrary to earlier observations, later studies revealed that during the early phase of EAU (postimmunization day 5) retinal $\mathrm{ONOO}^{-}$initiated damage occurred before there was any histologic or immunohistochemical evidence of macrophage infiltration or migration of retinal microglia $[6,15]$. These findings suggest that the innate immune response may play a role in inducing peroxynitrite-mediated oxidative stress during the early phase of EAU.

Prior to leukocyte infiltration during early EAU, selective peroxynitrite-mediated nitration of photoreceptor mitochondrial proteins occurs [17]. Our studies show that in the

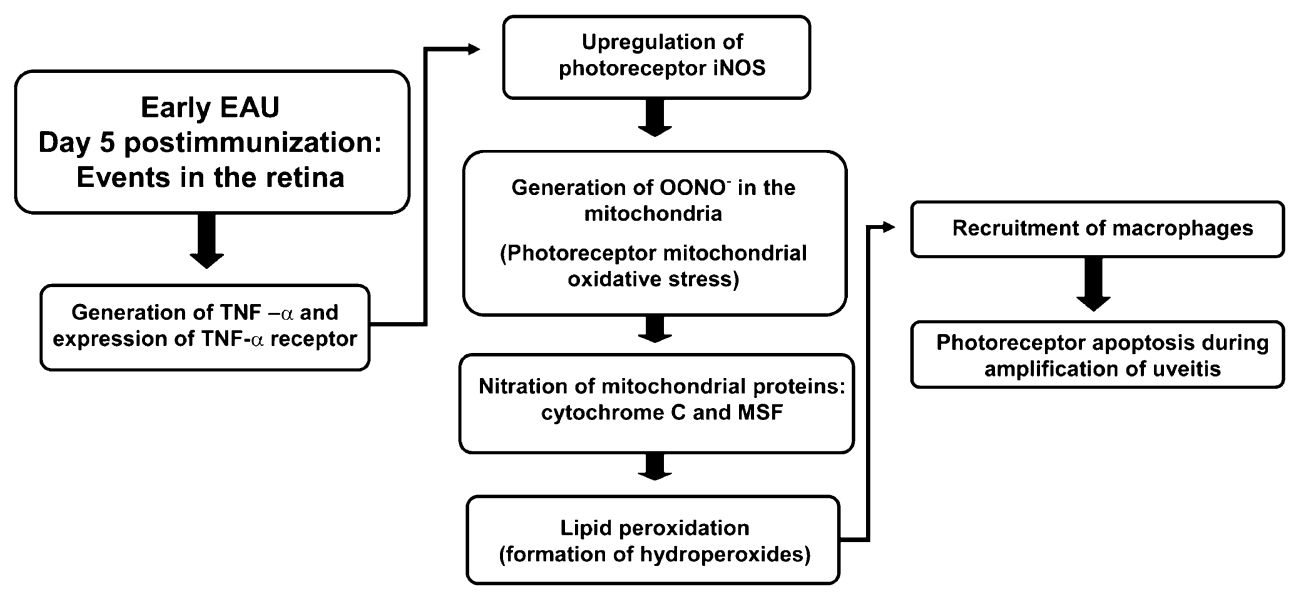

Fig. 2 During early EAU, inflammatory cytokines are upregulated, specifically tumor necrosis factor- $\alpha$ (TNF- $\alpha)$. TNF- $\alpha$ is a cytokine known to diffuse and upregulate inducible nitric oxide synthase in adjacent cells, resulting in the generation of $\mathrm{NO}$ and the subsequent formation of peroxynitrite $\left(\mathrm{ONOO}^{-}\right)$in the mitochondria which leads to mitochondrial oxidative stress. The nitration process takes place primarily in the photoreceptor inner segments, an area rich in mitochondria. Mitochondrial oxidative stress causes the initiation of lipid peroxidation and the formation of hydroperoxides; hydroperoxides, along with the nitrated mitochondrial proteins, function as chemotactic agents that amplify the inflammatory processes by recruiting macrophages, resulting in photoreceptor apoptosis 
early stages of EAU, mitochondria are the major source of $\mathrm{ONOO}^{-}$, and mitochondrial proteins are the prime target for inactivation, further implicating photoreceptor mitochondria as initiators of the proinflammatory response in early EAU [6]. Because of the selective nitration of photoreceptor mitochondrial proteins and the presence of peroxynitrite resulting from reactive oxidants and iNOS induced nitric oxide in the photoreceptor inner segment mitochondria, there appears to be photoreceptor mitochondrial oxidative stress during the early phase of EAU, before the infiltration of inflammatory cells; this stress is probably mediated by TNF- $\alpha$ induced iNOS and subsequent generation of peroxynitrite [18]. Although some observations suggest that during early EAU, a few activated $\mathrm{T}$ cells could infiltrate the retina and generate cytokines [18], a closer look at the innate immune response may give us a clearer picture about the cause behind mitochondrial oxidative stress. This review explores evidence for the development of mitochondrial oxidative stress from innate and adaptive immune effector processes of EAU and the stress-related retinal damage during autoimmune uveitis.

\section{Photoreceptor mitochondrial oxidative stress}

Mitochondrial DNA damage: an important marker for oxidative damage

Mitochondrial DNA (mtDNA) is more susceptible to oxidative damage than nuclear DNA (nDNA) because the former is in direct contact with mitochondrial produced reactive oxygen species; mtDNA are not protected by histones or other DNA-associated proteins [19-21]. In addition, the DNA repair machinery inherent in mitochondria is less efficient than nDNA [22, 23]. Therefore, in tissues rich in mitochondria, like the retina, the damaged or altered mtDNA serves as a reliable biomarker of oxidative stress.

Using a novel long quantitative polymerase chain reaction (QPCR technique), our laboratory demonstrated that mtDNA is damaged early in EAU (day 4 postimmunization). We also showed that in EAU, mitochondrial oxidative stress occurs before macrophage or other inflammatory cellular infiltration [7]. These findings support previous studies from our laboratory that determined that peroxynitrite-mediated nitration of photoreceptor mitochondrial proteins, as well as the presence of reactive oxidants and peroxynitrite, occurs in mitochondrial photoreceptors during early EAU [17, 18]. Oxidative stress appears to target the mitochondria as the original site of inflammation and thus damaged or altered mtDNA is a major target for oxidative damage.
iNOS-mediated oxidative stress results in the nitration of cytochrome $c$, a photoreceptor mitochondrial protein, which is then released into the cytosol; such cytochrome $c$ release is known to cause apoptosis $[6,18]$. Oxidative damage of mtDNA, if left unchecked, can lead to mitochondria dysregulation and cell death [24]. However, despite the presence of mtDNA damage, in our study apoptosis was not detected during early EAU (apoptosis occurred on day 12) [7]. Some of the reasons postulated by our laboratory for the lack of early cell death were that there may have been insufficient amounts of mtDNA damage to cause apoptotic cascades until day 12 or that protective mechanisms like heat shock proteins and crystallins, which are upregulated by oxidative stress, prevents photoreceptor apoptosis during early EAU by inhibiting components of the apoptotic cascade and functioning as protective and repair mechanisms $[3,7,25]$.

Differential expression of mitochondrial proteins and mitochondrial dysfunction

During early EAU, retinal DNA damage is restricted to the mitochondria; this suggests that mitochondrial oxidative stress plays a role in retinal damage [7]. Mitochondria are a source of reactive oxygen species and are the main target for stress-mediated damage. Oxidative stress can alter mitochondrial protein levels and consequently alter the functions of mitochondria in EAU. ATP synthases maintain mitochondrial membrane potentials and morphology [26]. Using 2D-DIGE, mass spectrometry by MALDI-TOF MS analysis, and reconfirming findings with Western bolt analysis, our laboratory found that during early EAU, there was a significant decrease in ATP synthase protein levels in retinal mitochondria; this suggests that decreased levels of cellular ATP and loss of ATP synthase activity corresponds to mitochondrial oxidative damage [3]. Moreover, the expression of aconitase, a sensitive marker of oxidative stress in mitochondria, was upregulated suggesting that mitochondrial oxidative stress affects its expression during early EAU [3].

Other proteins with altered expression due to early EAU were calretinin, mitochondrial aspartate aminotransferase, and malate dehydrogenase; we found decreased levels of calretinin, increased levels of mitochondrial aspartate aminotransferase, and decreased levels of malate dehydrogenase when compared to controls. These findings suggest mitochondrial dysfunction during early EAU. As we reported in an earlier study, damaged or altered mtDNA is a primary target for oxidative damage [7]. mtDNA damage causes amplification of oxidative stress by decreasing the expression of essential proteins for electron transport like malate dehydrogenase, leading to reactive oxygen species and mitochondrial dysregulation which will eventually cause apoptosis. 
The role of innate immunity in mediating photoreceptor mitochondrial oxidative stress

In early EAU, the mechanism that induces oxidative stress is still uncertain. Traditionally, it was believed that macrophages caused oxidative damage, but this seems unlikely since there is no histologic or immunohistochemical evidence of macrophage infiltration in the retina or uvea until days 11 to 12 whereas oxidative stress appears much earlier on days 5 to $7[6,15]$. In addition to macrophages, retinal microglia also exhibit phagocytic and proinflammatory pathogenic functions, however, they too do not appear to migrate towards the outer retina during early EAU. Our laboratory has previously reported that there is some evidence of adaptive immunity playing a role in the induction of oxidative stress; the presence of a few $\mathrm{CD}^{+}$ cells in the retina on day 5 postimmunization and real-time QPCR data showing an increase in CD28 transcripts in the retina, implicates the presence of activated $\mathrm{T}$ cells [18]. There is also a marked upregulation of inflammatory cytokines associated with the induction of oxidative stress, such as TNF- $\alpha$, iNOS, IFN $\gamma$, and IL $1 \alpha$ on day 5 postimmunization [18]. Such cytokines could be generated by the innate immune response in the retina; the number of activated $\mathrm{T}$ cell infiltration was minimal during early EAU. The presence of these cytokines coincides with the presence of mtDNA damage. TNF- $\alpha$ causes the upegulation of iNOS, which subsequently causes the production of nitric oxide and other oxidative factors that contribute to mitochondrial oxidative stress $[27,28]$. The early upregulation of TNF- $\alpha$, before the migration of retinal microglia and infiltration of macrophages, suggests that innate immunity could cause oxidative stress during early EAU.

The innate immune response may contribute to oxidative stress before the $\mathrm{T}$ cells have a chance to migrate into the retina. In a recent study, our laboratory used real-time PCR analysis to show that during early EAU, before leukocyte infiltration of the retina, there is increased expression of TNF- $\alpha$ and iNOS in nude mice compared with nonimmunized controls [4]. Since nude mice are deficient in T cells [29], the presence of these inflammatory cytokines confirms that innate immunity plays a role in the upregulation of cytokines.

Toll-like receptors (TLRs) are a group of transmembrane proteins that play an essential role in the innate immune response and in the upregulation of TNF- $\alpha[30,31]$. In vitro studies suggest that activation of TLR 4 can cause mitochondrial oxidative stress in the central nervous system and in the liver [32]. Similar mitochondrial oxidative stress is seen in photoreceptors in early EAU [5]. Our recent study confirmed the importance of TLR4 in the generation of proinflammatory cytokines crucial to the induction of mitochondrial oxidative stress; using real-time PCR and Western blot analysis, we found that TNF- $\alpha$ and iNOS were markedly downregulated in TLR4-deficient mice when compared with wild-type mice with EAU [4]. TLR4 deficiency was shown to attenuate iNOS gene expression and expression of this cytokine is known to cause mitochondrial oxidative stress [18]. We confirmed our finding using immunohistochemistry, which revealed that in addition to TNF- $\alpha$ and iNOS, photoreceptor mitochondrial oxidative stress was abrogated in the absence of TLR4, as observed with TLR4 knockout mice with EAU [4].

Our laboratory also revealed that during early EAU, the presence of TLR4 is crucial for the initiation of DNA oxidative damage. As stated in a previous study, damaged or altered mtDNA is a reliable biomarker of oxidative stress in tissues rich in mitochondria like the photoreceptor inner segments of the retina [7]. 8-hydroxy-2'-deoxyguanosine (8-OHdG), an oxidized form of deoxyguanosine, is a reliable indicator of DNA oxidative damage; during oxidative stress, 8 -OHdG levels increase preferentially in mitochondria as a result of the single-stranded nature of mtDNA, which makes it more susceptible to damage [33]. Our recent study showed that while there is substantial 8OHdG staining and thus mitochondrial DNA damage in wild-type mice with EAU, this is substantially reduced in TLR4 knockout mice (Fig. 3) [4]. The intense 8-OHdG staining we found in the photoreceptor inner segments of WT mice with EAU reinforces previous findings from our laboratory which indicate that in EAU retinas, photoreceptor cells are the primary site of oxidative damage $[12,18]$. Our recent studies also support previous reports that apoptosis is not a feature of early EAU; based on terminal deoxynucleotidyl transferase dUTP nick end labeling (TUNEL) staining, apoptotic cells were nor detected in WT or TLR4-deficient mice with EAU, suggesting the presence of a protective mechanism, such as $\alpha \mathrm{A}$ crystallin upregulation, in the photoreceptors that prevents photoreceptor apoptosis in early EAU $[4,7,25]$.

Although the presence of TLR4 is necessary for the upregulation of significant quantities of TNF- $\alpha$, iNOS, and 8-OHdG, other TLRs may also play a minor role in the induction of these gene products in early EAU since mild upregulation of these cytokines and mild oxidative DNA damage still occurs when TLR4 is not present. However, based on our findings, TLR4 has a functional significance in EAU and further study of TLR4 and innate immunity will enhance our understanding of the EAU pathway.

Photoreceptor oxidative damage in sympathetic ophthalmia

A recent study from our laboratory reveals that like EAU, sympathetic ophthalmia (SO) also results in photoreceptor mitochondrial oxidative stress and damage. SO is a diffuse bilateral granulomatous uveitis that is a potential complication of penetrating ocular injury to one eye and has the 


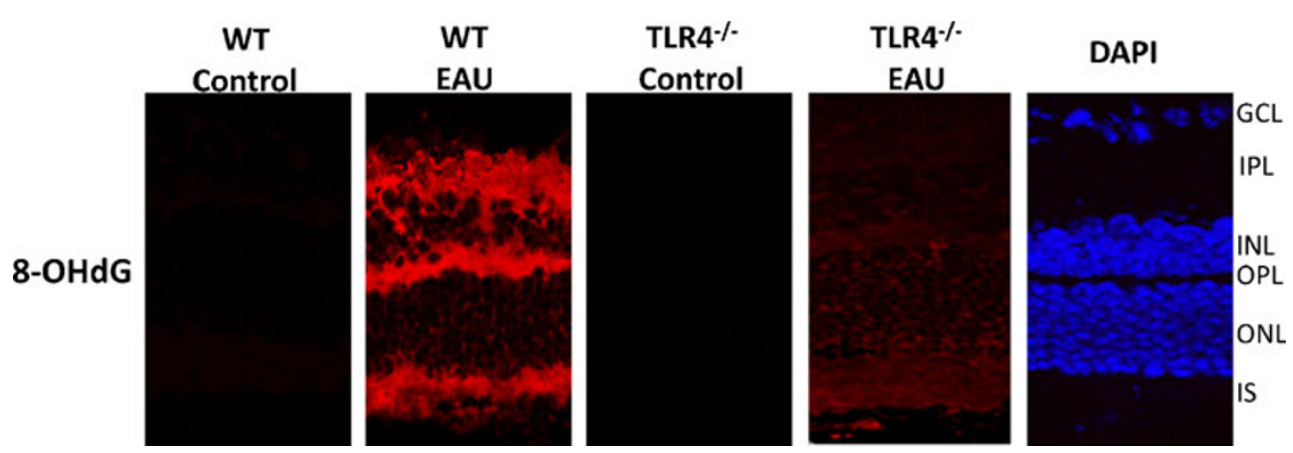

Fig. 3 Immunofluorescence localization of $8-\mathrm{OHdG}$ in the retinas of wild-type (WT; C57BL/6) mice and TLR4-deficient $\left(T L R 4^{-1-}\right)$ mice with EAU day 7 after immunization. Tissues were labeled using the polyclonal antibody against 8-OHdG and Texas Red dye-conjugated donkey anti-goat $\operatorname{IgG}$ as the primary and secondary antibodies respectively. No 8-OHdG staining was present in the nonimmunized (control) WT and TLR4 ${ }^{-1-}$ mice. In the WT mice with EAU, intense

potential to cause blindness to both eyes; in SO, leukocytic infiltration is seen in the uvea, but is absent in the choriocapillaries and retina $[34,35]$. Using immunofluorescent techniques, we found increased levels of TNF- $\alpha$ in the photoreceptor layer, which suggests that TNF- $\alpha$ plays a role in the induction of iNOS in SO [36]. At the inner segments of the photoreceptors, iNOS was colocalized with cytochrome $\mathrm{c}$, indicating its photoreceptor mitochondrial position. Thus, iNOS and nitrotyrosine were found in the mitochondria of the photoreceptors which clearly indicates the presence of photoreceptor mitochondrial oxidative stress in SO [11, 17, 18, 36]. The upregulation of both iNOS and nitrotyrosine occurred in the absence of CD3positive $\mathrm{T}$ or CD45-positive leukocyes in SO retinas. Our study showed apoptosis of few photoreceptors, but the sections of the eye also revealed the absence of extensive apoptosis in the photoreceptor cell layer, despite the presence of diffuse photoreceptor mitochondrial oxidative stress (Fig. 4) [36]. This observation suggests that like EAU, SO also has a protective mechanisms in place, like crystallins, to prevent apoptotis.

Like EAU, the mechanism behind SO photoreceptor oxidative stress remains unclear; since SO and EAU appear so similar, perhaps the same approach should be taken in studying both diseases. In SO photoreceptor damage and eventual blindness occurs in the absence of $\mathrm{T}$ cells and leukocyte infiltration in the retina $[34,36]$, suggesting that perhaps the role of innate immunity in SO needs to be looked at more closely.

\section{Conclusion}

During the early phase of EAU, photoreceptor mitochondrial oxidative stress occurred before there was any
8-OHdG staining was detected in the inner plexiform layer (IPL), inner nuclear layer $(I N L)$, outer plexiform layer $(O P L)$, and in the inner segments of the photoreceptor layer; such staining was significantly reduced in the outer plexiform layer and inner segments and was absent in the remainder of the retina in the TLR $4^{-/-}$mice during early EAU

histologic or immunohistochemical evidence of macrophage or other inflammatory cell infiltration. Oxidative stress appears to target the mitochondria of photoreceptors and the primary site of inflammation and mtDNA is targeted at the photoreceptors. However, despite the subsequent presence of mtDNA damage in the photoreceptors, apoptosis is not a characteristic of early EAU. Oxidative stress can also alter mitochondrial protein levels and consequently alter the functions of mitochondria in EAU. The presence of inflammatory cytokines, photoreceptor mitochondrial oxidative stress, and mtDNA damage

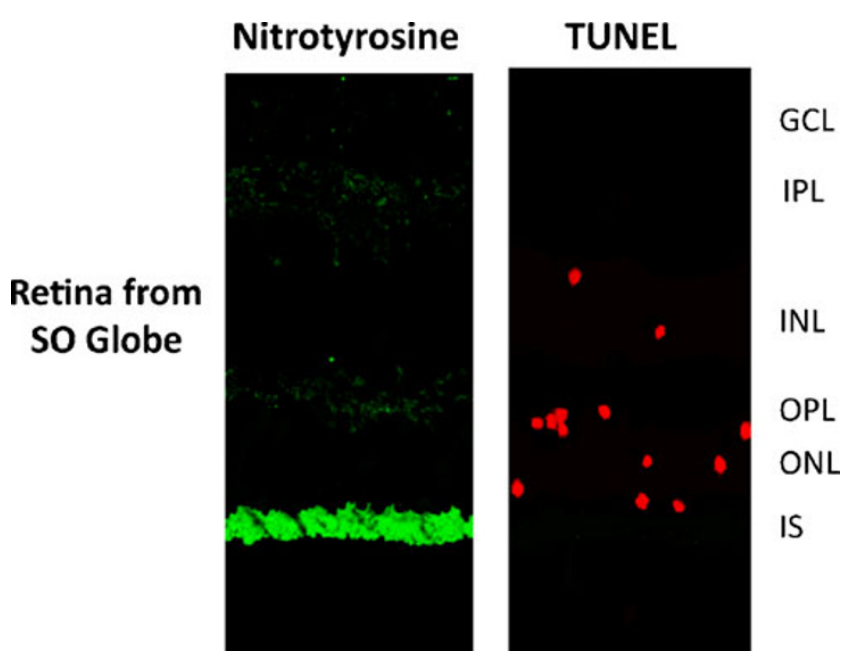

Fig. 4 Immunofluorescence colocalization of nitrotyrosine with apoptotic cells in retinas of patients with sympathetic ophthalmia (SO). SO globes were labeled using polyclonal rabbit antinitrotyrosine (primary antibody)and sheep-antirabbit IgG conjugated with flourescein (secondary antibody), followed by TUNEL staining using a TACS TdT Apoptosis Detection Kit (American Qualex). Nitrotyrosine was localized primarily in the photoreceptor inner segments (IS). Apoptotic cells were detected mainly in the outer nuclear layer $(O N L)$, with a few apoptotic cells in the inner nuclear layer $(I N L)$ in $\mathrm{SO}$ retinas 
before leukocyte infiltration during early EAU, suggests that the role of innate immunity, especially TLR4, has a functional significance in the EAU pathway. Recent studies show the similarities between EAU and SO; our results suggest that agents that may prevent photoreceptor mitochondrial oxidative stress and that may prevent photoreceptor apoptosis initiated by oxidative stress may help to prevent retinal damage and preserve vision in $\mathrm{SO}$ and perhaps other uveitic conditions.

Open Access This article is distributed under the terms of the Creative Commons Attribution Noncommercial License which permits any noncommercial use, distribution, and reproduction in any medium, provided the original author(s) and source are credited.

\section{References}

1. Rao NA (1990) Role of oxygen free radicals in retinal damage associated with experimental uveitis. Trans Am Ophthalmol Soc $88: 797-850$

2. Caspi RR (2003) Experimental autoimmune uveoretinitis in the rat and mouse. Curr Protoc Immunol Chapter 15:Unit 15.6. doi:10.1002/0471142735.im1506s53

3. Saraswathy S, Rao NA (2009) Mitochondrial proteomics in experimental autoimmune uveitis oxidative stress. Invest Ophthalmol Vis Sci 50(12):5559-5566. doi:10.1167/iovs.08-2842

4. Saraswathy S, Nguyen AM, Rao NA (2010) The role of tlr4 in photoreceptor \{alpha\}a crystallin upregulation during early experimental autoimmune uveitis. Invest Ophthalmol Vis Sci 51 (7):3680-3686. doi:10.1167/iovs.09-4575

5. Saraswathy S, Rao NA (2008) Photoreceptor mitochondrial oxidative stress in experimental autoimmune uveitis. Ophthalmic Res 40(3-4):160-164. doi:10.1159/000119869

6. Wu GS, Lee TD, Moore RE, Rao NA (2005) Photoreceptor mitochondrial tyrosine nitration in experimental uveitis. Invest Ophthalmol Vis Sci 46(7):2271-2281. doi:10.1167/iovs.04-1525

7. Khurana RN, Parikh JG, Saraswathy S, Wu GS, Rao NA (2008) Mitochondrial oxidative DNA damage in experimental autoimmune uveitis. Invest Ophthalmol Vis Sci 49(8):3299-3304. doi:10.1167/iovs.07-1607

8. Caspi R (2008) Autoimmunity in the immune privileged eye: Pathogenic and regulatory t cells. Immunol Res 42(1-3):41-50. doi:10.1007/s12026-008-8031-3

9. Luger D, Silver PB, Tang J, Cua D, Chen Z, Iwakura Y, Bowman EP, Sgambellone NM, Chan CC, Caspi RR (2008) Either a th17 or a th1 effector response can drive autoimmunity: Conditions of disease induction affect dominant effector category. J Exp Med 205(4):799-810. doi:10.1084/jem.20071258

10. Rao NA, Wu GS (2000) Free radical mediated photoreceptor damage in uveitis. Prog Retin Eye Res 19(1):41-68. doi:S13509462(99)00003-8[pii]

11. Wu GS, Zhang J, Rao NA (1997) Peroxynitrite and oxidative damage in experimental autoimmune uveitis. Invest Ophthalmol Vis Sci 38(7):1333-1339

12. Zhang J, Wu LY, Wu GS, Rao NA (1999) Differential expression of nitric oxide synthase in experimental uveoretinitis. Invest Ophthalmol Vis Sci 40(9):1899-1905

13. Goto H, Wu GS, Gritz DC, Atalla LR, Rao NA (1991) Chemotactic activity of the peroxidized retinal membrane lipids in experimental autoimmune uveitis. Curr Eye Res 10(11):1009-1014
14. Wu GS, Sevanian A, Rao NA (1992) Detection of retinal lipid hydroperoxides in experimental uveitis. Free Radic Biol Med 12 (1):19-27

15. Rao NA, Kimoto T, Zamir E, Giri R, Wang R, Ito S, Pararajasegaram G, Read RW, Wu GS (2003) Pathogenic role of retinal microglia in experimental uveoretinitis. Invest Ophthalmol Vis Sci 44(1):22-31

16. Saraswathy S, Wu G, Rao NA (2006) Retinal microglial activation and chemotaxis by docosahexaenoic acid hydroperoxide. Invest Ophthalmol Vis Sci 47(8):3656-3663. doi:10.1167/ iovs.06-0221

17. Ito $\mathrm{S}, \mathrm{Wu} \mathrm{GS}$, Kimoto T, Hisatomi T, Ishibashi T, Rao NA (2004) Peroxynitrite-induced apoptosis in photoreceptor cells. Curr Eye Res 28(1):17-24. doi:10.1076/ceyr.28.1.17.23488

18. Rajendram R, Saraswathy S, Rao NA (2007) Photoreceptor mitochondrial oxidative stress in early experimental autoimmune uveoretinitis. Br J Ophthalmol 91(4):531-537. doi:10.1136/ bjo. 2006.101576

19. Yakes FM, Van Houten B (1997) Mitochondrial DNA damage is more extensive and persists longer than nuclear DNA damage in human cells following oxidative stress. Proc Natl Acad Sci USA 94(2):514-519

20. Santos JH, Hunakova L, Chen Y, Bortner C, Van Houten B (2003) Cell sorting experiments link persistent mitochondrial DNA damage with loss of mitochondrial membrane potential and apoptotic cell death. J Biol Chem 278(3):1728-1734. doi:10.1074/jbc.M208752200

21. Ljungman M, Hanawalt PC (1992) Efficient protection against oxidative DNA damage in chromatin. Mol Carcinog 5(4):264-269

22. Croteau DL, Bohr VA (1997) Repair of oxidative damage to nuclear and mitochondrial DNA in mammalian cells. J Biol Chem 272(41):25409-25412

23. Mandavilli BS, Santos JH, Van Houten B (2002) Mitochondrial DNA repair and aging. Mutat Res 509(1-2):127-151. doi: S0027510702002208[pii]

24. Van Houten B, Woshner V, Santos JH (2006) Role of mitochondrial DNA in toxic responses to oxidative stress. DNA Repair Amst 5(2):145-152. doi:10.1016/j.dnarep.2005.03.002

25. Rao NA, Saraswathy S, Wu GS, Katselis GS, Wawrousek EF, Bhat S (2008) Elevated retina-specific expression of the small heat shock protein, alphaa-crystallin, is associated with photoreceptor protection in experimental uveitis. Invest Ophthalmol Vis Sci 49 (3):1161-1171. doi:10.1167/iovs.07-1259

26. Bornhovd C, Vogel F, Neupert W, Reichert AS (2006) Mitochondrial membrane potential is dependent on the oligomeric state of f1 f0-atp synthase supracomplexes. J Biol Chem 281(20):1399013998. doi:10.1074/jbc.M512334200

27. Borutaite V, Morkuniene R, Brown GC (1999) Release of cytochrome c from heart mitochondria is induced by high ca2+ and peroxynitrite and is responsible for $\mathrm{ca}(2+)$-induced inhibition of substrate oxidation. Biochim Biophys Acta 1453(1):41-48. doi: S0925-4439(98)00082-9[pii]

28. Moreno-Sanchez R (1985) Regulation of oxidative phosphorylation in mitochondria by external free ca2 + concentrations. J Biol Chem 260(7):4028-4034

29. Balciunaite G, Keller MP, Balciunaite E, Piali L, Zuklys S, Mathieu YD, Gill J, Boyd R, Sussman DJ, Hollander GA (2002) Wnt glycoproteins regulate the expression of foxn1, the gene defective in nude mice. Nat Immunol 3(11):1102-1108. doi:10.1038/ni850

30. Wang ZJ, Zhang FM, Wang LS, Yao YW, Zhao Q, Gao X (2009) Lipopolysaccharides can protect mesenchymal stem cells (mscs) from oxidative stress-induced apoptosis and enhance proliferation of mscs via toll-like receptor(tlr)-4 and pi3k/akt. Cell Biol Int 33 (6):665-674. doi:10.1016/j.cellbi.2009.03.006

31. Lee SJ, Lee S (2002) Toll-like receptors and inflammation in the cns. Curr Drug Targets Inflamm Allergy 1(2):181-191 
32. Suliman HB, Welty-Wolf KE, Carraway MS, Schwartz DA, Hollingsworth JW, Piantadosi CA (2005) Toll-like receptor 4 mediates mitochondrial DNA damage and biogenic responses after heat-inactivated e. Coli FASEB J 19(11):1531-1533. doi:10.1096/fj.04-3500fje

33. Ohtaki H, Takeda T, Dohi K, Yofu S, Nakamachi T, Satoh K, Hiraizumi Y, Miyaoka H, Matsunaga M, Shioda S (2007) Increased mitochondrial DNA oxidative damage after transient middle cerebral artery occlusion in mice. Neurosci Res 58(4):349355. doi:10.1016/j.neures.2007.04.005
34. Rathinam SR, Rao NA (2006) Sympathetic ophthalmia following postoperative bacterial endophthalmitis: A clinicopathologic study. Am J Ophthalmol 141(3):498-507. doi:10.1016/j.ajo.2005.10.047

35. Rao NA, Robin J, Hartmann D, Sweeney JA, Marak GE Jr (1983) The role of the penetrating wound in the development of sympathetic ophthalmia experimental observations. Arch Ophthalmol 101(1):102-104

36. Parikh JG, Saraswathy S, Rao NA (2008) Photoreceptor oxidative damage in sympathetic ophthalmia. Am J Ophthalmol 146 (6):866-875. doi:10.1016/j.ajo.2008.03.026, e862 